\title{
Set-membership Parity Space Approach for Fault Detection in Linear Uncertain Dynamic Systems
}

\author{
Joaquim Blesa*+, Vicenç Puig*+, Jordi Saludes*, Rosa M. Fernández-Cantí* \\ *Advanced Control Systems Group, Universitat Politècnica de Catalunya, \\ Pau Gargallo, 5, 08028 Barcelona, Spain. \\ + Institut de Robòtica i Informàtica Industrial (CSIC-UPC), \\ Carrer Llorenç Artigas, 4-6, 08222 Barcelona Spai \\ (e-mail: \{joaquim.blesa,vicenc.puig,jordi.saludes\}@upc.edu,rfernandez@tsc.upc.edu)
}

\begin{abstract}
In this paper, a set-membership parity space approach for linear uncertain dynamic systems is proposed. First, a set of parity relations derived from the parity space approach is obtained by means of a transformation derived from the system characteristic polynomial. As a result of this transformation parity relations can be expressed in regressor form. On one hand, this facilitates the parameter estimation of those relations using a zonotopic set-membership algorithm. On the other hand, fault detection is then based on checking, at every sample time, the non existence of a parameter value in the parameter uncertainty set such that the model is consistent with all the system measurements. The proposed approach will be applied to two examples a first illustrative case study based on a two-tank system and a more realistic case study based on the wind turbine FDI benchmark in order to evaluate its effectiveness.
\end{abstract}

Keywords: Fault Detection, Set-membership, Parity Space, Parity Equations, Zonotopes.

\section{INTRODUCTION}

Model-based fault diagnosis relies on analytical redundancy obtained by the use of mathematical models of the monitored system. Many approaches have been investigated and developed over the last few years (Gertler, 1998; Chen and Patton, 1999; Isermann, 2006; Blanke et al., 2006a). Reliability and performance of fault diagnosis algorithms depend on the quality of the model used. However, since modeling errors introduce uncertainty in the model, they interfere with the fault detection. A fault detection algorithm able to handle uncertainty is called robust and its robustness is the sensitivity to faults compared to the sensitivity to uncertainty (Chen and Patton, 1999). The effect of noise on the model-based fault detection is well understood using statistical approaches (Basseville and Nikiforov, 1993). However, in many situations the random nature of noise is 
unknown which makes the use of statistical methods difficult. This difficulty has led to develop an alternative description of the noise based on what is known as "unknown but bounded noise" description (Milanese et al., 1996). Moreover, not only noise but also modeling errors should be taken into account. Modeling error inclusion in statistical methods is far from being trivial. For all these reasons, these last years, research on robust fault detection methods that require only knowledge about bounds in noise and parameters (modelling errors) has been very active in the FDI community (Puig, 2010). These methods, known as set-membership, follow the passive robust approach (Chen and Patton, 1999) by enhancing the fault detection robustness at the decision-making stage using an adaptive threshold. This adaptive threshold is typically generated by considering the set of model trajectories that can be obtained by varying the uncertain parameters within their intervals (Puig, 2010).

This paper presents a set-membership parity space approach for uncertain linear dynamic systems as an alternative proposed in Ploix and Adrot (2006). This approach is based on a set of parity relations derived from the parity space approach using the Chow and Willsky scheme (Chow and Willsky, 1984). However, since the system is uncertain the decoupling from initial conditions is done using a symbolic approach in a similar way as Ploix and Adrot (2006). The main difference here is that the transformation is derived from the characteristic polynomial of the system state space representation. Using this idea, as discussed in (Ding, 2008), the set of residuals obtained are equivalent to primary parity equations approach proposed by Gertler (1998). Once the set of parity relations have been derived, they can be expressed in regressor form facilitating the parameter estimation and the fault detection test implementation. Parameters are estimated by the zonotope-based setmembership identification approach proposed by (Bravo et al., 2006). This enhances the results presented in Ploix and Adrot (2006) since no method for estimating parameter uncertainty is provided. On the other hand, after the model and its uncertainty has been calibrated using fault free data, fault detection is based on checking, at every sampling time, the non existence of a parameter value in the parameter uncertainty set such that model is consistent with all the system measurements using the socalled inverse test (Puig, 2006). This is different from Ploix and Adrot (2006) since the fault detection test was based on a linear approximation of the parity relations and checking whether zero is contained in the residual set (direct test).

A first academic example is used to illustrate in detail how the proposed approach works. Finally, a more realistic case study, based on the known FDI benchmark based on a wind turbine, is used to assess its performance.

The structure of this paper is the following: In Section 2, the problem statement is introduced. In Section 3, the residual transformation used to cancel the effect of initial conditions is introduced. Section 4 presents the fault detection methodology. 
Section 5 presents the model and uncertainty calibration approach. In Section 6, a two-tank system is used to illustrate in detail the presented methodology. Then, in Section 7, a more realistic case study based on the wind turbine FDI benchmark is used to assess the validity of the proposed approach. Finally, in Section 8, the main conclusions are drawn.

\section{PROBLEM STATEMENT}

\subsection{Problem set-up}

Let us consider that the system to be monitored can be described by the following uncertain state space model in discrete-time

$$
\begin{aligned}
& \boldsymbol{x}(k+1)=\mathbf{A}(\tilde{\boldsymbol{\theta}}) \boldsymbol{x}(k)+\mathbf{B}(\tilde{\boldsymbol{\theta}}) \boldsymbol{u}(k) \\
& \boldsymbol{y}(k)=\mathbf{C}(\tilde{\boldsymbol{\theta}}) \boldsymbol{x}(k)+\mathbf{D}(\tilde{\boldsymbol{\theta}}) \boldsymbol{u}(k)+\mathbf{E} \tilde{\boldsymbol{e}}(k)
\end{aligned}
$$

where

- $\boldsymbol{x}(k)$ is the state vector of dimension $n_{x} \times 1$. It is assumed that the initial state $\boldsymbol{x}(0)$ belongs to a known set $\boldsymbol{x}(0) \in \mathbb{X}_{0}$.

- $y(k)$ is the output vector of dimension $n_{y} \times 1$.

- $\boldsymbol{u}(k)$ is the input vector of dimension $n_{u} \times 1$.

- $\tilde{\boldsymbol{\theta}} \in \boldsymbol{\Theta}$ is the unknown time invariant real system parameter vector of dimension $n_{\theta} \times 1$ that belongs to a known set $\boldsymbol{\Theta}$.

- Matrices $\mathbf{A}(\tilde{\boldsymbol{\theta}}) \in \mathbb{R}^{n_{x} \times n_{x}}, \mathbf{B}(\tilde{\boldsymbol{\theta}}) \in \mathbb{R}^{n_{x} \times n_{u}}, \mathbf{C}(\tilde{\boldsymbol{\theta}}) \in \mathbb{R}^{n_{y} \times n_{x}}$, and $\mathbf{D}(\tilde{\boldsymbol{\theta}}) \in \mathbb{R}^{n_{y} \times n_{u}}$ are the system matrices and matrix $\mathbf{E} \in \mathbb{R}^{n_{y} \times n_{e}}$ the output noise matrix.

- $\tilde{\boldsymbol{e}}(k) \in \mathbb{E}$ is a vector of dimension $n_{e} \times 1$ that contains the additive error that is unknown but is assumed to be bounded by a set $\mathbb{E}$.

It is also assumed that the system defined by (1) is state observable.

Fault detection will be based on using an uncertain model of the system (1) that considers:

- model parameters $\boldsymbol{\theta}$ bounded by the known set $\boldsymbol{\Theta}$ ( i.e. $\boldsymbol{\theta} \in \boldsymbol{\Theta}$ ) that represents the uncertainty about the exact knowledge of the real system parameters $\tilde{\boldsymbol{\theta}}$.

- additive noise $\boldsymbol{e}(k)$ bounded by the known set $\mathbb{E}$ ( i.e. $\boldsymbol{e}(k) \in \mathbb{E}$ ) that represents the uncertainty about the exact knowledge of the real noise $\tilde{\boldsymbol{e}}(k)$.

Using this model, fault detection relies on generating a residual as a difference between the real and the model outputs defined 


$$
\boldsymbol{r}(k)=\boldsymbol{y}(k)-(\hat{\boldsymbol{y}}(k, \theta)+\mathbf{E} \boldsymbol{e}(k))
$$

where the model consist of an output estimation $\hat{\boldsymbol{y}}(k, \theta)$ that can be expressed in terms of the system initial condition $\boldsymbol{x}(0)$ as follows

$$
\hat{\boldsymbol{y}}(k, \theta)=\mathbf{C}(\theta) \boldsymbol{x}(k)+\mathbf{D}(\theta) \boldsymbol{u}(k)=\mathbf{C}(\theta)\left(\mathbf{A}^{k}(\theta) \boldsymbol{x}(0)+\sum_{i=0}^{k-1}\left(\mathbf{A}^{k-1-i}(\theta) \mathbf{B}(\theta) \boldsymbol{u}(i)\right)\right)+\mathbf{D}(\theta) \boldsymbol{u}(k)
$$

and a bounded error term $\mathbf{E} \boldsymbol{e}(k)$. For the sake of simplicity in notation the term $\hat{\boldsymbol{y}}(k, \theta)$ will be denoted $\hat{\boldsymbol{y}}(k)$ from now on. Notice that in order to know about the existence of a fault, this residual should be evaluated in a robust way, i.e. considering the model uncertainty and noise, as well the unknown initial conditions. Here, a passive robust approach will be used based on checking the consistency between the measurements and the model considering the uncertainty bounds.

\subsection{Consistency tests}

According to (Puig et al, 2006), there are two ways of checking consistency between the model (1) and the measurements: the direct and the inverse test. In the literature, most of the works dealing with uncertain models as (1) consider the direct test (Puig, 2010) following the seminal work of (Horak, 1998). Here, after revising the direct test, the inverse test will be proposed as more suitable for the suggested fault detection strategy proposed in later sections of the paper.

\subsubsection{Direct test}

Given a state space model (1) whose vector parameter $\boldsymbol{\theta}$ is considered invariant, the output measurement vector $\boldsymbol{y}(k)$ will be consistent at instant $k$ with the output predicted by the model, i.e., $y(k) \in[\underline{\hat{y}}(k), \overline{\hat{y}}(k)]$, when

$$
\mathbf{0} \in \boldsymbol{\Gamma}(i), \quad \forall i=1, \ldots, k
$$

where

$$
\Gamma(i)=\left\{\boldsymbol{r}(i) \mid \boldsymbol{e}(i) \in \mathbb{E}, \boldsymbol{x}(0) \in \mathbb{X}_{0}, \boldsymbol{\theta} \in \boldsymbol{\Theta}\right\}
$$

and $\boldsymbol{r}(i)$ is given by (2)-(3) and $\mathbf{0}$ is a vector $\left(n_{y} \times 1\right)$ of zeros $\mathbf{0}=(0 \cdots 0)^{t}$. The evaluation of the set (5) implies simulating the model (1) considering the uncertainty. This is in general a difficult task as discussed for example in (Puig, 2005) since if a recursive scheme is used based on approximating the output set with some approximating set as interval boxes or even zonotopes, it can lead to an explosion of uncertainty named as wrapping effect. Alternatively, if the computations are referred to the initial state as in (3) the computation of output set implies a complex optimization problem (Puig, 2003). Moreover as discussed in Puig (2006), since the residual parameter dependency is lost when evaluating (5), although (3) assumes invariant parameters, an artificially parameter variation is introduced. For this reason, a second test is proposed to be used in this paper. 


\subsubsection{Inverse test}

Given a state space model (1) whose vector parameter $\boldsymbol{\theta}$ is considered invariant, the output measurement vector $\boldsymbol{y}(k)$ will be consistent at instant $k$ with the output predicted by the model when

$$
\exists \boldsymbol{\theta} \in \boldsymbol{\Theta} \mid \mathbf{0} \in \boldsymbol{\Gamma}(i, \boldsymbol{\theta}), \quad \forall i=1, \ldots, k
$$

where

$$
\Gamma(i, \boldsymbol{\theta})=\left\{\boldsymbol{r}(i, \boldsymbol{\theta}) \mid \boldsymbol{e}(i) \in \mathbb{E}, \boldsymbol{x}(0) \in \mathbb{X}_{0}\right\}
$$

and $\mathbf{0}$ is a vector $\left(n_{y} \times 1\right)$ of zeros $\mathbf{0}=(0 \cdots 0)^{t}$.

At every instant $k$, the set of parameters consistent with the model and measurements is given by

$$
\mathbb{F}_{k}=\{\boldsymbol{\theta} \in \boldsymbol{\Theta} \mid \mathbf{0} \in \boldsymbol{\Gamma}(k, \boldsymbol{\theta})\}
$$

Then, the consistency test (6) can be formulated as

$$
\mathbf{F P S}_{k+1}=\bigcap_{i=1}^{k} \mathbb{F}_{i} \neq \varnothing
$$

where FPS $_{k+1}$ (Feasible Parameter Set at instant $k^{+1}$ ) contains all the vector parameters consistent at instant $k+1$ with previous data and can be computed in a recursive way as follows

$$
\mathbf{F P S}_{k+1}=\mathbf{F P S}_{k} \cap \mathbb{F}_{k}
$$

The computation of sets $\mathbb{F}_{k}$ and $\mathbf{F P S}_{k+1}$ is very complicated because the non linear dependence between residual and parameters (Jaulin et al., 2001). The aim of this paper is to arrive to a transformation of the residual (2) into one that allows to evaluate the consistent test (6) in an easy way.

\section{RESIDUAL TRANSFORMATION}

\subsection{Parity space approach}

The $n_{y}$ components of the residual $\boldsymbol{r}(k)$ defined in (2) can be computed separately as follows

$$
\boldsymbol{r}^{i}(k)=\boldsymbol{y}^{i}(k)-\hat{\boldsymbol{y}}^{i}(k)-\mathbf{E}^{i} \boldsymbol{e}(k) \quad i=1 \cdots n_{y}
$$

with

$$
\hat{\mathbf{y}}^{i}(k)=\mathbf{C}^{i}(\boldsymbol{\theta}) \boldsymbol{x}(k)+\mathbf{D}^{i}(\boldsymbol{\theta}) \boldsymbol{u}(k)=\mathbf{C}^{i}(\boldsymbol{\theta})\left(\mathbf{A}^{k}(\boldsymbol{\theta}) \boldsymbol{x}(0)+\sum_{i=0}^{k-1}\left(\mathbf{A}^{k-1-i}(\boldsymbol{\theta}) \mathbf{B}(\boldsymbol{\theta}) \boldsymbol{u}(i)\right)\right)+\mathbf{D}(\boldsymbol{\theta}) \boldsymbol{u}(k)
$$


where superscript $i$ in vectors $\boldsymbol{r}(k)$ and $\boldsymbol{y}(k)$ denotes the $i^{\text {th }}$ component of the vector and in matrices $\mathbf{C}(\theta), \mathbf{D}(\theta)$ and $\mathbf{E}$ denotes the $i^{\text {th }}$ row of the matrix.

Notice that residual components of $\boldsymbol{r}(k)$ depends on the unknown initial condition that introduces some additional uncertainty. A way to remove such a dependence is by introducing a transformation as suggested in the parity space approach introduced by Chow and Willsky (1984). However, in this case, the model (1) includes uncertainty in the parameters and such method does not apply directly. In (Ploix, 2006), a possible extended approach is proposed in the case the model is uncertain, but the computations needed are quite involved. Here, a different approach based on the equivalence that there exists between parity space approach and input/output models (Ding, 2008) is used.

\section{Proposition 1}

A transformed residual $\boldsymbol{r}_{w}(k)$, independent of the internal state $\boldsymbol{x}(k)$, can be found by using a transformation vector defined as follows

$$
\mathbf{w}(\theta)=\left(\begin{array}{llll}
\alpha_{0}(\theta) & \cdots & \alpha_{p-1}(\theta) & 1
\end{array}\right)
$$

where $\alpha_{0}(\theta), \cdots, \alpha_{p-1}(\theta), 1$ are the coefficients of the characteristic polynomial of the system (1):

$$
\operatorname{det}(\lambda \mathbf{I}-\mathbf{A}(\theta))=\lambda^{p}+\alpha_{p-1}(\theta) \lambda^{p-1}+\cdots+\alpha_{0}(\theta) .
$$

with $p=n_{X}$ and applied to the residual components of $\boldsymbol{r}(k)$ (10) as follows

with

$$
\mathbf{r}_{w}^{i}(k)=\mathbf{w}(\theta)\left(\mathbf{Y}^{i}(k)-\mathbf{T}^{i}(\theta) \mathbf{U}(k)-\mathbf{V}^{i}(k)\right)
$$

$$
\begin{aligned}
& \mathbf{Y}^{i}(k)=\left(\begin{array}{llll}
y^{i}(k-p) & \cdots & y^{i}(k-1) & y^{i}(k)
\end{array}\right)^{t} \\
& \mathbf{T}^{i}(\theta)=\left(\begin{array}{ccccc}
\mathbf{D}^{i}(\theta) & 0 & \cdots & 0 & 0 \\
\mathbf{C}^{i}(\theta) \mathbf{B}(\theta) & \mathbf{D}^{i}(\theta) & \cdots & 0 & 0 \\
\vdots & & \ddots & & \vdots \\
\mathbf{C}^{i}(\theta) \mathbf{A}^{p-1}(\theta) \mathbf{B}(\theta) & & \cdots & \mathbf{C}^{i}(\theta) \mathbf{B}(\theta) & \mathbf{D}^{i}(\theta)
\end{array}\right) \\
& \mathbf{U}(k)=\left(\begin{array}{llll}
\boldsymbol{u}(k-p) & \cdots & \boldsymbol{u}(k-1) & \boldsymbol{u}(k))^{\mathrm{t}}
\end{array}\right. \\
& \mathbf{V}^{i}(k)=\left(\begin{array}{ccc}
\mathbf{E}^{i} & 0 & 0 \\
0 & \ddots & 0 \\
0 & 0 & \mathbf{E}^{i}
\end{array}\right)(\boldsymbol{e}(k-p) \quad \cdots \quad \boldsymbol{e}(k-1) \quad \boldsymbol{e}(k))^{\mathrm{t}}
\end{aligned}
$$

\section{Proof}

Measurement output vector $\mathbf{Y}^{i}(k)$ in (14) can be computed using model (1) and $p+1$ step-ahead predictions, leading to

$$
\mathbf{Y}^{i}(k)=\mathbf{O}^{i}(\theta) \boldsymbol{x}(k)+\mathbf{T}^{i}(\theta) \mathbf{U}(k)+\mathbf{V}^{i}(k)
$$

with 


$$
\mathbf{O}^{i}(\theta)=\left(\begin{array}{c}
\mathbf{C}^{i}(\theta) \\
\mathbf{C}^{i}(\theta) \mathbf{A}(\theta) \\
\vdots \\
\mathbf{C}^{i}(\theta) \mathbf{A}^{p}(\theta)
\end{array}\right)
$$

$\mathbf{T}^{i}(\theta), \mathbf{U}(k)$ and $\mathbf{V}^{i}(k)$ defined as in (15)-(17). Then, if the left and right parts of (17) are multiplied by the vector $\mathbf{w}(\theta)$ defined as in (15) yields

$$
\mathbf{w}(\theta) \mathbf{Y}^{i}(k)=\mathbf{w}(\theta)\left(\mathbf{O}^{i}(\theta) \boldsymbol{x}(k)+\mathbf{T}^{i}(\theta) \mathbf{U}(k)+\mathbf{V}^{i}(k)\right)
$$

and by applying the Cayley-Hamilton theorem to Eq. (19) follows that

$$
\mathbf{w}(\theta) \mathbf{O}^{i}(\theta)=\alpha_{0}(\theta) \mathbf{C}^{i}(\theta)+\alpha_{1}(\theta) \mathbf{C}^{i}(\theta) \mathbf{A}(\theta)+\cdots+\mathbf{C}^{i}(\theta) \mathbf{A}^{p}(\theta)=0
$$

Thus, Eq. (19) results in

$$
\mathbf{w}(\theta) \mathbf{Y}^{i}(k)=\mathbf{w}(\theta)\left(\mathbf{T}^{i}(\theta) \mathbf{U}(k)+\mathbf{V}^{i}(k)\right)
$$

that allows to derive the residual defined in (10). This completes the proof

The system model (1) can be expressed in input/output form as follows:

$$
\hat{\mathbf{y}}(k)=\mathbf{M}\left(q^{-1}, \boldsymbol{\theta}\right) \mathbf{u}(k)=\frac{\mathbf{N}_{y u}\left(q^{-1}, \boldsymbol{\theta}\right)}{D_{y u}\left(q^{-1}, \boldsymbol{\theta}\right)} \mathbf{u}(k)
$$

where $\mathbf{M}\left(q^{-1}, \boldsymbol{\theta}\right)=\mathbf{C}(\boldsymbol{\theta})(q \mathbf{I}-\mathbf{A}(\boldsymbol{\theta}))^{-1} \mathbf{B}(\boldsymbol{\theta})+\mathbf{D}(\boldsymbol{\theta})$ is the transfer function matrix, and $\mathbf{N}_{y u}\left(q^{-1}, \boldsymbol{\theta}\right)$ and $D_{y u}\left(q^{-1}, \boldsymbol{\theta}\right)$ contain the numerator and denominator polynomials, respectively. For $i^{\text {th }}$ output and $j^{\text {th }}$ input, (22) reduces so a single transfer function whose denominator is the characteristic polynomial (12): $D_{y u}\left(q^{-1}, \boldsymbol{\theta}\right)=1+\alpha_{p-1}(\theta) q^{-1}+\cdots+\alpha_{0}(\theta) q^{-p}$. Thus, equivalently, the residual given by Eq. (13) can also be expressed in transfer function form using the shift operator $q^{-1}$ as follows:

$$
\mathbf{r}_{w}^{i}(k)=\mathbf{G}^{i}\left(q^{-1}, \boldsymbol{\theta}\right) \mathbf{u}(k)+\mathbf{H}^{i}\left(q^{-1}, \boldsymbol{\theta}\right)\left(\mathbf{y}^{i}(k)-\boldsymbol{E}^{i} \boldsymbol{e}(k)\right)
$$

where:

$$
\begin{gathered}
\mathbf{H}^{i}\left(q^{-1}, \theta\right)\left(\mathbf{y}^{i}(k)-\boldsymbol{E}^{i} \boldsymbol{e}(k)\right)=\mathbf{w}(\theta) \mathbf{Y}^{i}(k)=\mathbf{w}(\boldsymbol{\theta})\left[\begin{array}{c}
\mathbf{I} q^{-p} \\
\vdots \\
\mathbf{I}
\end{array}\right]\left(\mathbf{y}^{i}(k)-\boldsymbol{E}^{i} \boldsymbol{e}(k)\right) \\
\mathbf{G}^{i}\left(q^{-1}, \boldsymbol{\theta}\right) \mathbf{u}(k)=\mathbf{w}(\boldsymbol{\theta}) \mathbf{T}^{i}(\boldsymbol{\theta}) \mathbf{U}(k)=\mathbf{w}(\boldsymbol{\theta}) \mathbf{T}^{i}(\boldsymbol{\theta})\left[\begin{array}{c}
\mathbf{I} q^{-p} \\
\vdots \\
\mathbf{I}
\end{array}\right] \mathbf{u}(k)
\end{gathered}
$$


Remark 1: Notice that $\mathbf{G}^{i}\left(q^{-1}, \boldsymbol{\theta}\right)$ and $\mathbf{H}^{i}\left(q^{-1}, \boldsymbol{\theta}\right)$ can be straightforwardly related with polynomials $\mathbf{N}_{y u}\left(q^{-1}, \boldsymbol{\theta}\right)$ and $\mathrm{D}_{y u}\left(q^{-1}, \boldsymbol{\theta}\right)$ of the input-output model of the system (18) taking into account that (23) can be generated directly from (18) following the parity equation approach in MA (Moving Average) form (Gertler, 1998). Thus

$$
\begin{gathered}
\mathbf{G}^{i}\left(q^{-1}, \boldsymbol{\theta}\right)=\mathbf{N}_{y u}^{i}\left(q^{-1}, \boldsymbol{\theta}\right)=\mathbf{w}(\boldsymbol{\theta})\left[\begin{array}{c}
\mathbf{I} q^{-p} \\
\vdots \\
\mathbf{I}
\end{array}\right] \\
\mathbf{H}^{i}\left(q^{-1}, \boldsymbol{\theta}\right)=\mathbf{I}-\mathrm{D}_{y u}\left(q^{-1}, \boldsymbol{\theta}\right)=\mathbf{w}(\boldsymbol{\theta}) \mathbf{T}^{i}(\boldsymbol{\theta})\left[\begin{array}{c}
\mathbf{I} q^{-p} \\
\vdots \\
\mathbf{I}
\end{array}\right]
\end{gathered}
$$

\subsection{Relation with the interval observer approach}

Given the uncertain system (1), an interval observer with Luenberger structure to monitor can be written as (Puig, 2005):

$$
\begin{aligned}
& \hat{\mathbf{x}}(k+1, \boldsymbol{\theta})=\mathbf{A}(\boldsymbol{\theta}) \hat{\mathbf{x}}(k, \boldsymbol{\theta})+\mathbf{B}(\boldsymbol{\theta}) \mathbf{u}(k)+\mathbf{L}(\mathbf{y}(k)-\hat{\mathbf{y}}(k)-\mathbf{E e}(k)) \\
& =\mathbf{A}_{o}(\boldsymbol{\theta}) \hat{\mathbf{x}}(k, \boldsymbol{\theta})+\mathbf{B}_{o}(\boldsymbol{\theta}) \mathbf{u}(k)+\mathbf{L y}(k)-\mathbf{L E e}(k) \\
& \hat{\mathbf{y}}(k)=\mathbf{C}(\boldsymbol{\theta}) \hat{\mathbf{x}}(k, \boldsymbol{\theta})+\mathbf{D}(\boldsymbol{\theta}) \mathbf{u}(k)
\end{aligned}
$$

where $\mathbf{A}_{o}(\boldsymbol{\theta})=\mathbf{A}(\boldsymbol{\theta})-\mathbf{L C}(\boldsymbol{\theta}), \mathbf{B}_{o}(\boldsymbol{\theta})=\mathbf{B}(\boldsymbol{\theta})-\mathbf{L D}(\boldsymbol{\theta})$ and $\hat{\mathbf{x}}(k, \boldsymbol{\theta})$ is the estimated system space-state vector and for a given value of $\theta \in \Theta$.

The observer gain matrix $\mathbf{L} \in \mathbb{R}^{n x \times n y}$ is designed to stabilise the matrix $\mathbf{A}_{o}(\boldsymbol{\theta})=\mathbf{A}(\boldsymbol{\theta})-\mathbf{L C}(\boldsymbol{\theta})$ and to guarantee a desired performance regarding fault detection for all $\theta \in \Theta$ using LMI pole placement (Chilali et al., 1996). The effect of the uncertain parameters $\theta$ on the observer temporal response $\hat{\mathbf{y}}(k)$ will be bounded using an interval as in Section 2.2.

Remark 2: Notice that according to Meseguer et al. (2010), two particular cases could be distinguished in the interval observer according to the observer gain $\mathbf{L}$. First, the observer becomes a simulator when $\mathbf{L}=\mathbf{0}$ since the observer eigenvalues are those ones of the plant, but it can only be used when the system is stable. This corresponds to the model expressed as in (1). Second, the observer becomes a predictor when the observer gain $\left(\mathbf{L}=\mathbf{L}_{\mathbf{p}}\right){ }^{*}$ is selected such that all the observer eigenvalues are at the origin (“deadbeat observer”) and corresponds to the parity space approach expressed as in (10) (Chow et al., 1984).

Remark 3: Notice that following Ding (2008), the residual generated by the observer scheme (24) can be expressed in parity space approach form by expressing the observer (24) in canonical observable form and selecting the observer gain as follows

\footnotetext{
${ }^{*}$ When $\boldsymbol{C}$ has an inverse, the observer structure forces the predictor approach to satisfy $\boldsymbol{L}_{\boldsymbol{p}} \boldsymbol{C}=\boldsymbol{A}$.
} 


$$
\mathbf{L}=-\left[\begin{array}{c}
\alpha_{0}(\theta) \\
\vdots \\
\alpha_{p-1}(\theta)
\end{array}\right]-\left[\begin{array}{c}
\alpha_{0}^{o}(\theta) \\
\vdots \\
\alpha_{p-1}^{o}(\theta)
\end{array}\right]
$$

where $\alpha_{0}(\theta), \cdots, \alpha_{p-1}(\theta)$ are the coefficients of the system characteristic polynomial (12), while $\alpha_{0}^{o}(\theta), \cdots, \alpha_{p-1}^{o}(\theta)$ are those of the observer characteristic polynomial fixed by the observer poles (dynamics). Then, the vector transformation equivalent to (12) that allows obtaining a transformed residual (10) when the observer (24) is used is given by:

$$
\mathbf{w}(\theta)=\left(\begin{array}{llll}
\alpha_{0}^{o}(\theta) & \cdots & \alpha_{0}^{p-1}(\theta) & 1
\end{array}\right)
$$

Thus, only in the special case that the observer poles are placed in the origin (dead-beat observer), the residuals provided by parity space approach provided by the system transformation (12) will the same as the ones using the observer in input/output form (Puig et al., 2008)

$$
\hat{\mathbf{y}}(k)=\mathbf{G}^{i}\left(q^{-1}, \theta\right) \mathbf{u}(k)+\mathbf{H}^{i}\left(q^{-1}, \theta\right)(\mathbf{y}(k)-\mathbf{E e}(k))
$$

where

$$
\begin{aligned}
& \mathbf{H}^{i}\left(q^{-1}, \boldsymbol{\theta}\right)=\mathbf{I}-\mathbf{C}^{i}\left(q \mathbf{I}-\mathbf{A}_{o}(\boldsymbol{\theta})\right)^{-1} \mathbf{L} \\
& \mathbf{G}^{i}\left(q^{-1}, \boldsymbol{\theta}\right)=\mathbf{C}^{i}\left(q \mathbf{I}-\mathbf{A}_{o}(\boldsymbol{\theta})\right)^{-1} \mathbf{B}_{o}(\boldsymbol{\theta})+\mathbf{D}^{i}
\end{aligned}
$$

In this case

$$
\mathbf{L}=-\left[\begin{array}{c}
\alpha_{0}(\theta) \\
\vdots \\
\alpha_{p-1}(\theta)
\end{array}\right]
$$

and according to Remark 3, this corresponds to the case that $\mathbf{L}=\mathbf{L}_{\mathbf{p}}$, i.e., the observer leads to a residual equal to the parity space approach (23) and to the MA parity equations obtained from the input-output model (22) as shown by Gertler (1998).

Remark 4: According to (Patton et al., 1991), the predictor obtained forcing $\mathbf{L}=\mathbf{L}_{\mathbf{p}}$ is the predictor of minimum order (i.e. , the dead-beat observer) which can only indicate a fault for a minimum time period given by the system order.

\section{PARITY SPACE EQUIVALENCE}

\subsection{Parity space}

From (21), a model in regressor form for every output can be extracted 


$$
y_{i}(k)=\varphi_{i}(k) \xi+n_{i}(k) \quad i=1 \cdots n_{y}
$$

where

- $\boldsymbol{\varphi}_{i}(k)$ is the regressor vector of dimension $1 \times n_{\xi}$ which can contain any function of inputs $\boldsymbol{u}(k)$ and outputs $\boldsymbol{y}(k)$.

- $\xi \in \Xi$ is the parameter vector of dimension $n_{\xi} \times 1$.

- $\Xi$ is the set that bounds parameter $\xi$ values.

- $n_{i}(k)$ is the additive error bounded by a constant $\left|n_{i}(k)\right| \leq \varepsilon_{i}$.

Remark 5: Dependence of parameter vector $\xi$ and additive error $n_{i}(k)$ in (31) with respect to the parameter vector $\theta$ and additive error $e_{i}(k)$ in (1) can be obtained analytically from (18).

Remark 6: In the same way, set $\boldsymbol{\Xi}$ and bounds $\varepsilon_{i}$ can be related to sets $\boldsymbol{\Theta}$ and $\mathbb{E}$.

The $n_{y}$ individual models (31) can be expressed in a compact form as a Multiple Input and Multiple Output (MIMO) model

$$
\boldsymbol{y}(k)=\boldsymbol{\Phi}(k) \xi+\boldsymbol{n}(k)
$$

where

- $\boldsymbol{\Phi}(k)$ is the regressor matrix of dimension $n_{y} \times n_{\xi}$ that contains the regressor vectors.

- $\boldsymbol{n}(k)$ is a vector of dimension $n_{y} \times 1$ that contains the additive errors.

\subsection{Consistency test}

Now considering model (31), $\mathbb{F}_{k}$ defined in (8) can be expressed as the intersection of $n_{y}$ strips

$$
\mathbb{F}_{k}=\mathbf{F}_{1, k} \cap \cdots \cap \mathbf{F}_{n_{y}, k}
$$

where

$$
\mathbf{F}_{i, k}=\left\{\xi \in \mathbb{R}^{n_{\xi}} \mid y_{i}(k)-\varepsilon_{i} \leq \boldsymbol{\varphi}_{i}(k) \xi \leq y_{i}(k)+\varepsilon_{i}\right\}
$$

Then, the $\mathbf{F P S}_{k+1}$ that contains all the vector parameters $\xi$ consistent with previous data at instant $k+1$ is a polytope that can be defined as 


$$
\mathbf{F P S}_{k+1}=\left\{\xi \in \mathbb{R}^{n_{\xi}} \mid \begin{array}{c}
y_{1}(i)-\varepsilon_{1} \leq \boldsymbol{\varphi}_{1}(i) \xi \leq y_{1}(i)+\varepsilon_{1} \\
\vdots \\
y_{n_{y}}(i)-\varepsilon_{n_{y}} \leq \boldsymbol{\varphi}_{n_{y}}(i) \xi \leq y_{n_{y}}(i)+\varepsilon_{n_{y}}
\end{array} \quad, \forall i=1, \ldots, k\right\}
$$

And, consistency test (9) consists in determining the feasibility of the following linear problem.

$$
\mathbf{P}_{k} \xi \leq \mathbf{b}_{k}
$$

with

$$
\begin{aligned}
& \mathbf{P}_{k}=\left(\begin{array}{lllllll}
-\boldsymbol{\varphi}_{1}(1) & \boldsymbol{\varphi}_{1}(1) & \cdots & \boldsymbol{\varphi}_{n y}(1) & -\boldsymbol{\varphi}_{1}(2) & \cdots & \boldsymbol{\varphi}_{n y}(k)
\end{array}\right)^{\mathrm{t}}
\end{aligned}
$$

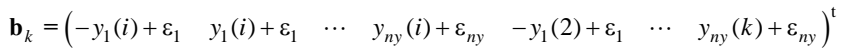

If the linear consistency problem (35) is unfeasible, then condition (9) is not fulfilled and a fault is detected.

The main drawback of this fault detection test is that the number of constraints (given by $2 k n_{y}$ ) in the linear problem (35) grows with time (data) (Blesa et al. 2012). In order to avoid dealing with the exact description of the FPS , existing algorithms usually approximate the FPS using inner/outer simpler shapes as boxes, parallelotopes, ellipsoids or zonotopes (Milanese et al., 1996). The approximation set is called Approximated Feasible Parameter Set (AFPS ).

Outer approximation algorithms find the parameter set AFPS that approximates the polytope FPS with a simpler shape (box, parallelotope, ellipsoid or zonotope) of minimum volume such that FPS $\subseteq$ AFPS . This kind of algorithms usually implies an excessive computational cost and recursive forms have been proposed in order to reduce the computational cost. For instance, the one proposed by Vicino and Zappa (1996) in case of using parallelotopes and the one proposed by Bravo et al. (2006) in case of using zonotopes. In order to maximize the accuracy in fault detection, outer approximations try to minimize their volume.

Inner approximation algorithms find the parameter set AFPS of maximum volume such that $\mathbf{A F P S} \subseteq$ FPS . In particular, in this work the recursive zonotope-based outer algorithm proposed by Bravo et al. (2006) will be used that allows computing

$$
\mathbf{A}_{\text {out }} \mathbf{F P S}_{k+1} \supseteq \mathbf{A}_{\text {out }} \mathbf{F P S}_{k} \cap \mathbb{F}_{k}
$$

This approach has already been used for fault detection purposes in Blesa et al. (2011a).

Alternatively, consistency test can be carry out by means of the direct test defined in (4) with

where

$$
\boldsymbol{\Gamma}(k)=\left(r_{1}(k), \ldots, r_{\text {ny }}(k)\right)
$$

$$
r_{i}(k)=y_{i}(k)-\boldsymbol{\varphi}_{i}(k) \xi-n_{i}(k) \quad i=1 \cdots n_{y}
$$


Given model (1) and taken into account transformation (23), the structure of model (32) defined by matrix $\boldsymbol{\Phi}(k)$ and vector $\xi$ can be obtained directly. Then, the problem of model calibration boils down in determining bounds for additive error $\varepsilon_{i}$ $\left(i=1 \cdots n_{y}\right)$ and parameter set $\Xi$.

Additive error bounds $\varepsilon_{i}$ can be obtained easily from bounded set $\mathbb{E}$ because the dependence of $\boldsymbol{n}(k)$ with $\boldsymbol{e}(k)$ is linear. But, on the other hand, as the relation between parameters $\xi$ and $\theta$ is nonlinear, the problem of finding $\Xi$ is very difficult to tackle directly from model (1).

In the following, a methodology that describes how to find a set $\boldsymbol{\Xi}$ consistent with data is presented.

Let us consider a sequence of $M$ regressor matrix values $\boldsymbol{\Phi}(k)$ and output measurements $\boldsymbol{y}(k)$ in a fault free scenario, the model of the system to be monitored parameterized as in (32) and the parameter set $\Xi$ described by a zonotope centered in a nominal model:

$$
\Xi=\xi^{0} \oplus \mathbf{H} \mathbb{B}^{n}=\left\{\xi^{0}+\mathbf{H z}: \mathbf{z} \in \mathbb{B}^{n}\right\}
$$

where

- $\xi^{0} \in \mathbb{R}^{n_{\xi}}$ is the center of the zonotope that corresponds to the nominal model.

- $\mathbf{H} \in \mathbb{R}^{n_{\xi} \times n}$ is the shape of the zonotope (usually $n \geq n_{\xi}$ and as the bigger $n$ is, the more complicated relations between uncertainty component parameters can be taken into account).

- $\mathbb{B}^{n}$ is a unitary box composed by $n$ unitary $(\mathbb{B}=[-1,1])$ interval vectors.

- $\oplus$ denotes the Minkowski sum.

The aim is to estimate a nominal parameter vector $\xi^{0}$ and their uncertainty (model set) defined by the matrix $\mathbf{H}$ in such a way that all measured data in a fault free scenario satisfy feasibility of linear problem (35).

Let us consider an initial set defined by physical limits

$$
\Xi_{\text {ini }}=\xi_{\text {ini }}^{0} \oplus \mathbf{H}_{\text {ini }} \mathbb{B}^{n}=\left\{\xi_{\text {ini }}^{0}+\mathbf{H}_{\text {ini }} \mathbf{z}: \mathbf{z} \in \mathbb{B}^{n}\right\}
$$

and by using the outer algorithm (37) proposed in Bravo et al. (2006) with initial condition

$$
\mathbf{A}_{\text {out }} \mathbf{F P S}_{0}=\boldsymbol{\Xi}_{\text {ini }}
$$

the set $\mathbf{A}_{\text {out }} \mathbf{F P S}_{M+1}$, resulting from applying (37) to all the data, defines the set $\boldsymbol{\Xi}$. 


\section{ILLUSTRATIVE EXAMPLE}

\subsection{Description of the system}

A two-tank process will be used to illustrate the results presented in this paper. A schematic diagram of the system is shown in

Figure 1 . The process inputs are $v_{1}$ and $v_{2}$ (input voltages to the pumps) and the outputs are the tank levels $h_{1}$ and $h_{2}$. The equations that describe the system are

$$
\begin{aligned}
& \frac{d h_{1}}{d t}=-\frac{a_{1}}{A_{1}} \sqrt{2 g h_{1}}+\frac{a_{2}}{A_{1}} \sqrt{2 g h_{2}}+\frac{\mathrm{k}_{1}}{A_{1}} v_{1} \\
& \frac{d h_{2}}{d t}=-\frac{a_{2}}{A_{2}} \sqrt{2 g h_{2}}+\frac{\mathrm{k}_{2}}{A_{2}} v_{2}
\end{aligned}
$$

where $a_{1}=a_{2}=0.071 \mathrm{~cm}^{2}, A_{1}=A_{2}=28 \mathrm{~cm}^{2}, g=981 \mathrm{~cm} / \mathrm{s}^{2}, k_{1}=0.231 \mathrm{~cm}^{3} / V s, k_{2}=0.21 \mathrm{~cm}^{3} / V s$ and assumed constants.

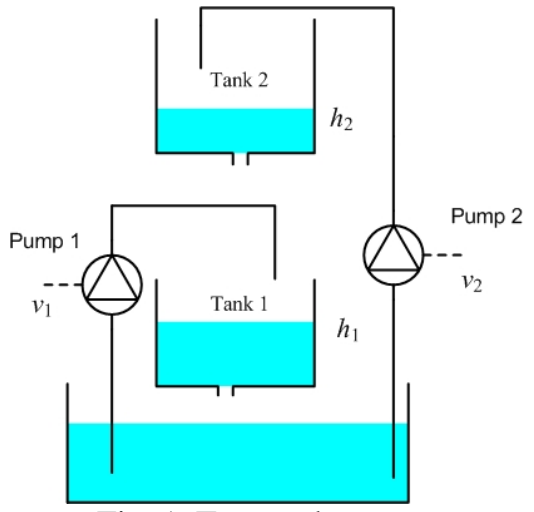

Fig. 1. Two-tank system

Eq. (30) can be discretised by the Euler method with sampling time $\Delta t=1 \mathrm{~s}$ and linearised in an operating point defined by $h_{1}^{0}$ and $h_{2}^{0}$. Then, (43) can be expressed as in (1) through the following parameterisation

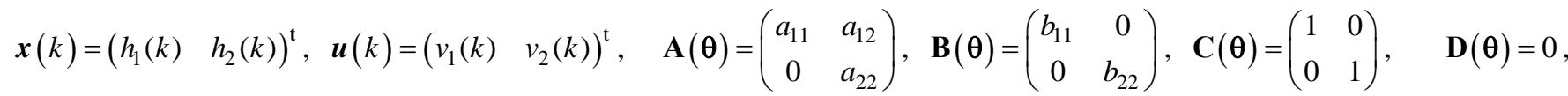
$\mathbf{E}=\left(\begin{array}{ll}1 & 0 \\ 0 & 1\end{array}\right)$ and $\boldsymbol{e}(k)=\left(e_{1}(k) \quad e_{2}(k)\right)^{\mathrm{t}}$

where

$a_{11}=1-\frac{a_{1}}{A_{1}} \sqrt{\frac{2 g}{h_{1}^{0}}}, a_{12}=\frac{a_{2}}{A_{1}} \sqrt{\frac{2 g}{h_{2}^{0}}}, \quad a_{22}=1-\frac{a_{2}}{A_{2}} \sqrt{\frac{2 g}{h_{2}^{0}}}$,

$b_{11}=\frac{k_{1}}{A_{1}}, b_{22}=\frac{k_{2}}{A_{2}}$. Variables $e_{1}(k), e_{2}(k)$ contain the additive errors due to discretisation, linearisation and sensor noises that are bounded by constants $\sigma_{1}$ and $\sigma_{2}$, respectively.

\subsection{Residual transformation}

Residuals (10) of model (30) can be transformed into residuals (13) with 


$$
\mathbf{w}(\theta)=\left(\begin{array}{lll}
a_{11} a_{22} & -\left(a_{11}+a_{22}\right) & 1
\end{array}\right)
$$

Then, the state space representation of model (43) is equivalent to MIMO model (31) with

$$
\begin{gathered}
\boldsymbol{\Phi}^{\mathrm{t}}(k)=\left(\begin{array}{cc}
y_{1}(k-1) & y_{2}(k-1) \\
-y_{1}(k-2) & -y_{2}(k-2) \\
v_{1}(k-1) & 0 \\
-v_{1}(k-2) & 0 \\
v_{2}(k-2) & 0 \\
0 & v_{2}(k-1) \\
0 & -v_{2}(k-2)
\end{array}\right) \quad \xi=\left(\begin{array}{c}
a_{11}+a_{22} \\
a_{11} a_{22} \\
b_{1} \\
b_{1} a_{22} \\
a_{12} b_{2} \\
b_{2} \\
b_{2} a_{11}
\end{array}\right) \\
\boldsymbol{n}(k)=\left(\begin{array}{c}
e_{1}(k)-\left(a_{11}+a_{22}\right) e_{1}(k-1)+\left(a_{11} a_{22}\right) e_{1}(k-2) \\
e_{2}(k)-\left(a_{11}+a_{22}\right) e_{2}(k-1)+\left(a_{11} a_{22}\right) e_{2}(k-2)
\end{array}\right)
\end{gathered}
$$

and where $\varepsilon_{1}=\sigma_{1}\|\mathbf{w}(\theta)\|_{1}$ and $\varepsilon_{2}=\sigma_{2}\|\mathbf{w}(\theta)\|_{1}$.

\subsection{Model identification}

In order to apply the calibration method presented in Section 5, a fault free scenario rich enough from the identification point of view has been simulated around an operating point given by $h_{1}^{0}=12.48 \mathrm{~cm}$ and $h_{2}^{0}=1.52 \mathrm{~cm}$.

Considering maximum variation levels $\Delta h_{1}=0.5 \mathrm{~cm}, \Delta h_{2}=0.5 \mathrm{~cm}$ and the physical limitation in parameters $\theta$ the initial set

(41) is computed as

$$
\xi_{\text {ini }}^{0}=\left(\begin{array}{c}
1.873 \\
0.876 \\
0.083 \\
0.075 \\
0.005 \\
0.048 \\
0.046
\end{array}\right), \mathrm{H}_{\text {ini }}=10^{-2} \operatorname{diag}\left(\begin{array}{c}
1.678 \\
1.621 \\
0 \\
0.134 \\
0.077 \\
0 \\
0.003
\end{array}\right)
$$

Then, applying (37) to the fault free identification data, the consistent set (40) obtained is defined by

$$
\begin{aligned}
& \xi^{0}=\left(\begin{array}{lllllll}
1.873 & 0.876 & 0.083 & 0.075 & 0.0046 & 0.048 & 0.046
\end{array}\right)^{\mathrm{t}} \\
& \mathrm{H}_{0}=10^{-2}\left(\begin{array}{ccccccc}
0.045 & 1.634 & 0 & 0.0331 & -0.018 & 0 & 0 \\
0 & 1.621 & 0 & 0 & 0 & 0 & 0 \\
0 & 0 & 0 & 0 & 0 & 0 & 0 \\
0 & 0 & 0 & 0.134 & 0 & 0 & 0 \\
0 & 0 & 0 & 0 & 0.077 & 0 & 0 \\
0 & 0 & 0 & 0 & 0 & 0 & 0 \\
0 & 0 & 0 & 0 & 0 & 0 & 0.003
\end{array}\right)
\end{aligned}
$$


Figure 2 shows the initial set $\Xi_{\text {ini }}$ (defined by $\xi_{\text {ini }}^{0}$ and $H_{\text {ini }}$ ) and the consistent set $\Xi$ (defined by $\xi^{0}$ and $\mathrm{H}_{0}$ ) obtained after the identification procedure.

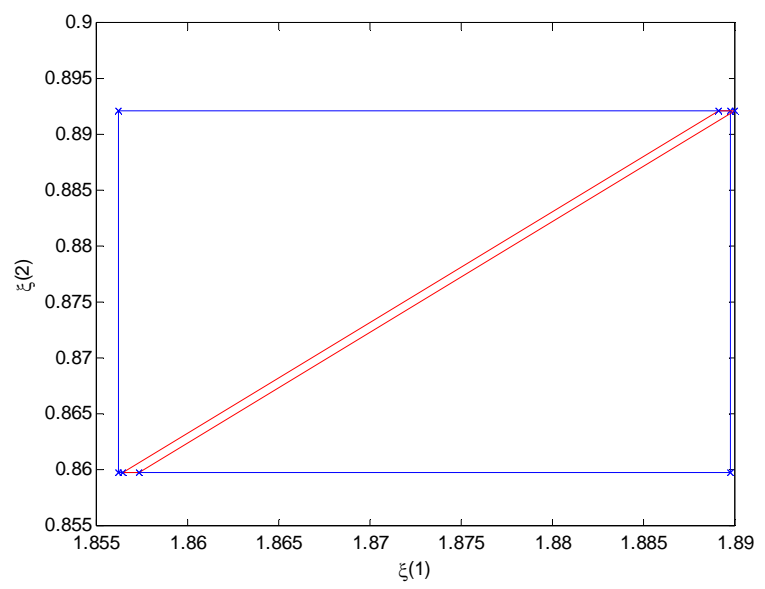

Fig. 2. $\xi(1)-\xi(2)$ Projection of the initial and identified consistency sets: $\Xi_{\text {ini }}$ (blue box) and $\Xi$ (red zonotope).

\subsection{Consistency test}

In order to illustrate the consistency test defined in Section 4 in several fault scenarios, two different kinds of faults have been considered: additive faults (in input and output sensors: $\boldsymbol{f}_{\boldsymbol{u}}$ and $\boldsymbol{f}_{\boldsymbol{y}}$ ) and multiplicative faults (in parameters: $\boldsymbol{f}_{\theta}$ ).

In the following, two fault scenarios have been simulated in the operation point presented in Figure 3 and the results of the fault detection procedure are shown.
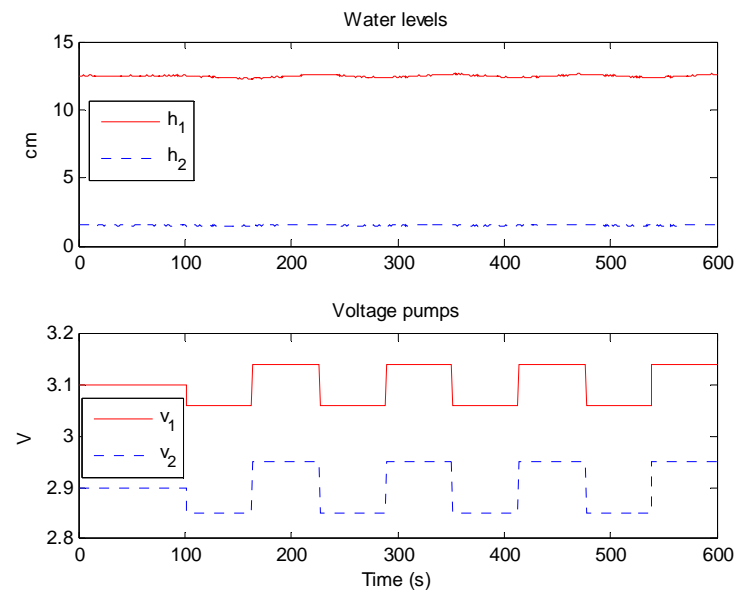

Fig. 3. No faulty scenario

Fault scenario1: “ $y_{1}$ sensor additive fault of $f_{y_{1}}=0.25 \mathrm{~cm}$ at $t=250 \mathrm{~s}$ ”

Figure 4 shows the behaviour of the consistency test defined in (37) in fault scenario 1. In this figure, it can be noticed that the strip $\mathbf{F}_{1,250}$ (given by output 1 at $t=250$ s) does not intersect with the outer zonotope that bounds the consistent set. Thus, the fault is detected at the same instant. 


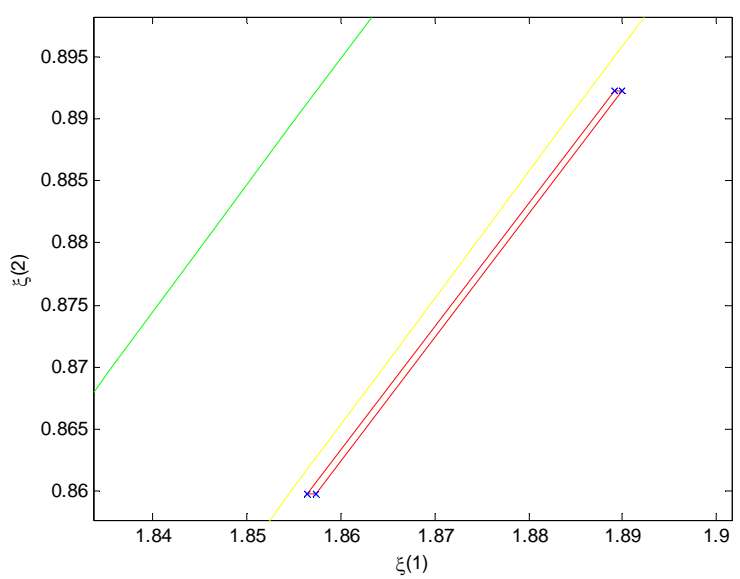

Fig. 4. Consistency test in fault scenario 1. Outer zonotope (in red) and stripe $\mathbf{F}_{1,250}$ (bounded by the green and yellow lines) at instant $t=250$ s.

Fault scenario 2: “ $a_{1}$ multiplicative fault of $f_{a_{1}}=-0.053 \mathrm{~cm}^{2}$ at $t=400 \mathrm{~s}$ ”

Figure 5 shows the behaviour of the consistency test defined in (37) in fault scenario 2. In this figure, it can be noticed that the strip $\mathbf{F}_{1,400}$ (given by output 1 at $t=400 s$ ) does not intersect with the outer zonotope that bounds the consistent set. Thus, the fault is detected at the same instant.

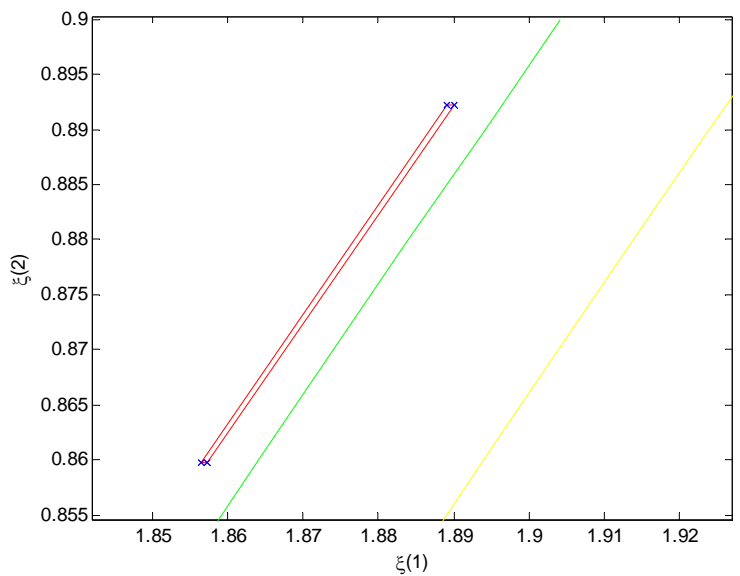

Fig. 5. Consistency test in fault scenario 2. Outer zonotope (in red) and stripe $\mathbf{F}_{1,400}$ (bounded by the green and yellow lines) at instant $t=400$ s.

\section{CASE STUDY: WIND TURBINE}

\subsection{Description}

This example is based on the realistic FDI case study proposed in "Wind turbine Benchmark" proposed in (Odgaard et al., 2013) where the challenge is to detect and to isolate a set of pre-defined faults. 
From the subsystems (Blade \& Pitch system, Drive Train, Generator \& Converter and Controller) model presented in (Odgaard et al., 2013), the structural analysis proposed by Staroswiecki, presented in (Blanke et al., 2006a), can be applied using the SaTool (Blanke et al., 2006b). The variables considered as known are the measured outputs $\left(v_{w}, \omega_{r}, \omega_{g}, \beta_{1}, \beta_{2}, \beta_{3}, \tau_{g}\right.$ and $P_{g}$ ) and controlled inputs ( $\tau_{g r}$ and $\left.\beta_{g r}\right) . \tau_{r}$ is estimated from the wind velocity $v_{w}$ using Eq. (4) in (Odgaard et al., 2013).

\subsection{Residual generation and fault isolation}

According to Blesa et al. (2011,b), after applying structural analysis (Blanke et al., 2006) with the aid of the SaTool (Blanke and Lorentz, 2006) to the set of equations provided in Odgaard et al. (2013) and considering the set of redundant sensors $\left(\omega_{r_{-} m 2}, \omega_{g_{-} m 2}, \beta_{1 \_m 2}, \beta_{2 \_m 2}\right.$ and $\left.\beta_{3 \_m 2}\right)$, the following set of residuals can be generated

$$
\begin{aligned}
& r_{1}=\omega_{r_{-} m 1}-\omega_{r_{-} m 2} \\
& r_{2}=\omega_{r_{-} m 2}-\hat{\omega}_{r}\left(\omega_{r_{-} m 2}, \tau_{r}, \tau_{g_{-} m}\right) \\
& r_{3}=\omega_{g_{-} m 1}-\omega_{g_{-} m 2} \\
& r_{4}=\omega_{g_{-} m 2}-\hat{\omega}_{g}\left(\omega_{g_{-} m 2}, \tau_{r}, \tau_{g_{-} m}\right) \\
& r_{5}=\beta_{11_{-} m 1}-\beta_{1_{-} m 2} \\
& r_{6}=\beta_{1 \_m 2}-\hat{\beta}_{1}\left(\beta_{1-m 2}, \beta_{r}\right) \\
& r_{7}=\beta_{2 \_m 1}-\beta_{2 \_m 2} \\
& r_{8}=\beta_{2_{-} m 2}-\hat{\beta}_{2}\left(\beta_{2 \_m 2}, \beta_{r}\right) \\
& r_{9}=\beta_{3_{-} m 1}-\beta_{3_{-} m 2} \\
& r_{10}=\beta_{3_{-} m 2}-\hat{\beta}_{3}\left(\beta_{3_{-} m 2}, \beta_{r}\right) \\
& r_{11}=\tau_{g_{-} m}-\hat{\tau}_{g}\left(\tau_{g_{-} m}, \tau_{g r}\right) \\
& r_{12}=p_{g_{-} m}-\hat{p}_{g}\left(\omega_{g_{-} m 2}, \tau_{g_{-} m}\right)
\end{aligned}
$$

where residuals $r_{2}, r_{4}, r_{6}, r_{8}, r_{10}, r_{11}$ and $r_{12}$ follows from analytical redundancy relations $e_{1}, e_{2}, e_{3}, e_{4}, e_{5}, e_{6}$ and $e_{7}$. On the hand, the remaining residuals are generated directly comparing the redundant sensors.

Fault isolation consists in identifying the faults affecting the system. As proposed in classic FDI books (Gertler, 1998; Isermann, 2006), faults can be isolated on the basis of fault signatures generated by a fault detection test applied componentwise to each single residual

$$
\phi_{i}(k)=\left\{\begin{array}{ccc}
0 & \text { if } & r_{i}(k) \quad \text { is consistent } \\
1 & \text { if } & r_{i}(k) \text { is not consistent }
\end{array}\right.
$$

producing an observed fault signature $\phi(k)$ :

$$
\phi(k)=\left(\phi_{1}(k), \phi_{2}(k), \cdots, \phi_{n_{\phi}}(k)\right)
$$


The observed fault signature is thus supplied to the fault isolation module that has knowledge of the binary relation between the considered fault hypothesis set $\boldsymbol{f}(k)=\left\{f_{1}(k), f_{2}(k), \cdots, f_{n_{f}}(k)\right\}$ and the observed fault signature $\phi(k)$. This relation is stored in what is called the theoretical binary fault signature matrix (FSM). An element $F S M_{i ; j}$ of this matrix is equal to 1 if the fault hypothesis $f_{j}(k)$ is expected to affect the residual $r_{i}(k)$, that is, the related fault signal $\phi_{i}(k)$ is equal to 1 when this fault affects the monitored system. Otherwise, the element $F S M_{i ; j}$ has a zero value. Analysing the effect of each of the faults proposed in (Odgaard et al., 2013) in the previous residuals (45) using fault sensitivity analysis (Gertler, 1998), the fault signature matrix presented in Table 2 is obtained.

\begin{tabular}{l|l|l|l|l|l|l|l|l|} 
& $f_{1}$ & $f_{2}$ & $f_{3}$ & $f_{4}$ & $f_{5}$ & $f_{6}$ & $f_{7}$ & $f_{8}$ \\
\hline$r_{1}$ & & & & $\mathrm{x}$ & $\mathrm{x}$ & & & \\
\hline$r_{2}$ & $\mathrm{x}$ & $\mathrm{x}$ & $\mathrm{x}$ & & $\mathrm{x}$ & $\mathrm{x}$ & $\mathrm{x}$ & $\mathrm{x}$ \\
\hline$r_{3}$ & & & & & $\mathrm{x}$ & & & \\
\hline$r_{4}$ & $\mathrm{x}$ & $\mathrm{x}$ & $\mathrm{x}$ & & $\mathrm{x}$ & $\mathrm{x}$ & $\mathrm{x}$ & $\mathrm{x}$ \\
\hline$r_{5}$ & $\mathrm{x}$ & & & & & & & \\
\hline$r_{6}$ & $\mathrm{x}$ & & & & & & & \\
\hline$r_{7}$ & & $\mathrm{x}$ & & & & & & \\
\hline$r_{8}$ & & $\mathrm{x}$ & & & & $\mathrm{x}$ & & \\
\hline$r_{9}$ & & & $\mathrm{x}$ & & & & & \\
\hline$r_{10}$ & & & $\mathrm{x}$ & & & & $\mathrm{x}$ & \\
\hline$r_{11}$ & & & & & & & & $\mathrm{x}$ \\
\hline$r_{12}$ & & & & & $\mathrm{x}$ & & & \\
\hline
\end{tabular}

Table 2: Theoretical signature matrix where " $x$ " denotes that a residual is sensitive to a fault

The accepted standard procedure involves finding a perfect matching between the observed fault signature and one of the theoretical fault signatures (Gertler, 1998). However, as discussed in (Tornil et al., 2013), this reasoning is not appropriate in an unknown but bounded context as the one considered in this paper. Here, the isolation scheme suggested by (Tornil et al., 2013) is used that, under single-fault assumption, considers that the fault that is actually present in the system has to affect all the residuals that have been found inconsistent according to the observed fault signature. The robustness of the fault isolation method can be improved adding active FDI approaches that provides extra excitation by means of auxiliary input signals in order to activate the largest possible number of residuals when a fault has been occurred and thus increasing the distinguishability between faults (Tabatabaeipour 2013).

\subsection{Residual transformation and calibration}

In order to apply the fault detection method proposed in this paper, the whole wind turbine system can be divided in 5 subsystems that can be described by the state space models 


$$
\begin{aligned}
& \boldsymbol{x}_{i}(k+1)=\mathbf{A}_{i}(\theta) \boldsymbol{x}_{i}(k)+\mathbf{B}_{i}(\theta) \boldsymbol{u}_{i}(k) \\
& \boldsymbol{y}_{i}(k)=\mathbf{C}_{i}(\boldsymbol{\theta}) \boldsymbol{x}_{i}(k)+\mathbf{D}_{i}(\theta) \boldsymbol{u}_{i}(k)+\mathbf{E}_{i} \boldsymbol{e}_{i}(k)
\end{aligned} \quad i=1, \cdots, 5
$$

with $\mathbf{D}_{i}(\theta)=0$ and $\mathbf{E}_{i}=\mathbf{I}, i=1, \cdots, 5$

where the five subsystems are:

Pitch subsystems 1,2 and 3: related to residuals $r_{6}, r_{8}$ and $r_{10}$ of Eq. (45) and can be modeled by

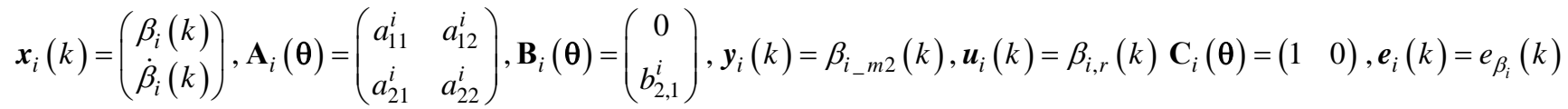

$i=1,2,3$

where coefficients of matrices $\mathbf{A}_{i}(\theta)$ and $\mathbf{B}_{i}(\theta) \quad(i=1,2,3)$ can be computed discretizing Eq. (6) in (Odgaard et al., 2013).

Drive train subsystem: related to residuals $r_{2}$ and $r_{4}$ of Eq. (45) and can be modelled by

$$
\begin{array}{ll}
\boldsymbol{x}_{4}(k)=\left(\begin{array}{c}
\omega_{r}(k) \\
\omega_{g}(k) \\
\theta_{\Delta}(k)
\end{array}\right), \quad \mathbf{A}_{4}(\theta)=\left(\begin{array}{ccc}
a_{1,1}^{4} & a_{1,2}^{4} & a_{1,3}^{4} \\
a_{2,1}^{4} & a_{2,2}^{4} & a_{2,3}^{4} \\
a_{3,1}^{4} & a_{3,2}^{4} & a_{3,3}^{4}
\end{array}\right) \quad \mathbf{B}_{4}(\theta)=\left(\begin{array}{cc}
b_{1,1}^{4} & 0 \\
0 & b_{2,2}^{4} \\
0 & 0
\end{array}\right) \quad \boldsymbol{u}_{4}(k)=\left(\begin{array}{c}
\tau_{r}(k) \\
\tau_{g_{-} m}(k)
\end{array}\right), \quad \boldsymbol{y}_{4}(k)=\left(\begin{array}{c}
\omega_{r_{-} m 2}(k) \\
\omega_{g_{-} m 2}(k)
\end{array}\right), \\
\mathbf{C}_{4}(\theta)=\left(\begin{array}{lll}
1 & 0 & 0 \\
0 & 1 & 0
\end{array}\right), \boldsymbol{e}_{4}(k)=\left(\begin{array}{c}
e_{\omega_{r}}(k) \\
e_{\omega_{g}}(k)
\end{array}\right),
\end{array}
$$

where $\theta_{\Delta}$ is an inner state of the drive train subsystem and coefficients of matrices $\mathbf{A}_{4}(\theta)$ and $\mathbf{B}_{4}(\theta)$ can be computed discretizing Eq. (7) in (Odgaard et al., 2013).

Converter subsystem: related to residual $r_{11}$ of Eq. (45) and can be modelled by

$$
\boldsymbol{x}_{5}(k)=\tau_{g}(k), \mathbf{A}_{5}(\theta)=a_{1,1}^{5}, \mathbf{B}_{5}(\theta)=b_{1,1}^{5}, \boldsymbol{u}_{5}(k)=\tau_{g, r}(k), \boldsymbol{y}_{5}(k)=\tau_{g_{-} m}(k), \mathbf{C}_{5}(\theta)=1 \quad \boldsymbol{e}_{2}(k)=e_{\tau g}(k)
$$

where coefficients $a_{1,1}^{5}$ and $b_{1,1}^{5}$ can be computed discretizing Eq. (9) in (Odgaard et al., 2013).

Following the methodology described in Section 3, residuals $r_{2}, r_{4}, r_{6}, r_{8}, r_{10}$ and $r_{11}$ are derived from the transformation of (48) and following the methodology described in Section 5 the obtained parity equations are calibrated in a fault free scenario. Finally, the consistency of the obtained residuals can be evaluated on-line in order to detect faults using consistency test defined in Section 4. 
Regarding static residuals $r_{1}, r_{3}, r_{5}, r_{7}$ and $r_{10}$ and from redundant measurements and $r_{12}$ from static power equation in (Odgaard et al., 2013), additive error bounds $\varepsilon_{i}$ have been calculated as the maximum value of the residuals in a fault free scenario.

$$
\varepsilon_{i}=\max _{k=1, \ldots, M}\left|r_{i}(k)\right| \quad i=\{1,3,5,7,9,12\}
$$

where $M$ is the length of the fault free scenario.

Once the additive error bounds have been calibrated, the consistency of static residuals has been calculation of fault signature $\phi_{i}(k)$ associated to residuals $r_{1}, r_{3}, r_{5}, r_{7}, r_{10}$ and $r_{12}$ can be computed by

$$
\phi_{i}(k)=\left\{\begin{array}{l}
0 \text { if }\left|r_{i}(k)\right| \leq \varepsilon_{i} \\
1 \text { if }\left|r_{i}(k)\right|>\varepsilon_{i}
\end{array}\right.
$$

\subsection{Results}

The proposed fault detection method and the isolation method described in previous section has been applied to the FDI Benchmark described in Odgaard et al. (2013) with satisfactory results in all the proposed fault scenarios except fault scenario 6 whose fault effects are very small. Table 3 summarizes the fault detection and isolation results using the proposed method and the results obtained with two other set-membership methods: the state space set-membership FDI method proposed in Tabatabaeipour et al. 2012 and the interval observer FDI approach proposed in Blesa et al. 2014.

\begin{tabular}{|c|c|c|c|c|}
\hline $\begin{array}{l}\text { Fault } \\
\text { scenario }\end{array}$ & $\begin{array}{l}\text { Fault appearance } \\
\text { time }\end{array}$ & $\begin{array}{l}\text { Fault diagnosis time } \\
\text { Proposed method }\end{array}$ & $\begin{array}{l}\text { Fault diagnosis time } \\
\text { Tabatabaeipour et al 2012 }\end{array}$ & $\begin{array}{c}\text { Fault diagnosis time } \\
\text { Blesa et al 2014 }\end{array}$ \\
\hline 1 & $2000 \mathrm{~s}$ & $2000.04 \mathrm{~s}$ & $2000.01 \mathrm{~s}$ & $2000.03 \mathrm{~s}$ \\
\hline 2 & $2300 \mathrm{~s}$ & $2307.38 \mathrm{~s}$ & $2304.21 \mathrm{~s}$ & $2300.06 \mathrm{~s}$ \\
\hline 3 & $2600 \mathrm{~s}$ & $2600.03 \mathrm{~s}$ & $2600.01 \mathrm{~s}$ & $2600.03 \mathrm{~s}$ \\
\hline 4 & $1500 \mathrm{~s}$ & $1500.08 \mathrm{~s}$ & $1500.05 \mathrm{~s}$ & $1500.03 \mathrm{~s}$ \\
\hline 5 & $1000 \mathrm{~s}$ & $1000.01 \mathrm{~s}$ & $1000.01 \mathrm{~s}$ & $1000.03 \mathrm{~s}$ \\
\hline 6 & $2900 \mathrm{~s}$ & $2977.63 \mathrm{~s}$ & $2951.69 \mathrm{~s}$ & $3511.60 \mathrm{~s}$ \\
\hline 7 & $3500 \mathrm{~s}$ & $3547.11 \mathrm{~s}$ & $3524.83 \mathrm{~s}$ & $3800.04 \mathrm{~s}$ \\
\hline 8 & $3800 \mathrm{~s}$ & $3800.01 \mathrm{~s}$ & $3800.01 \mathrm{~s}$ & $\mathrm{~s}$ \\
\hline
\end{tabular}

Table 3: Fault detection and isolation behaviour for the set of considered faults in the WT FDI competition using the proposed method and two other set-membership methods

The behaviour of the proposed method presents similar results for Faults 1,2,3,4,5 and 8 compared to those of the two other methods and slightly worse in case of the Faults 6 and 7 . The poor behaviour of the parity space approach in case of these two last faults can be explained because the combination of two effects: First the parity space behaves as a dead-beat observer (see Remark 4). This implies that after a number of samples (related to the order of the system) after the fault appearance, the

\footnotetext{
${ }^{\dagger}$ Increasing $10 \%$ the magnitude of this fault to the one proposed in Odgaard et al. (2013)
} 
residual tends to be small even the fault still is present. Second, faults 6 and 7 present a smooth and slow dynamic effect in the residuals. However, as also noticed in (Ploix and Adrot, 2006), the parity space:

- presents a lower computational cost compared to the state estimation methods (as the ones used for comparison in Table 3) and does not require to tune an observer gain.

- $\quad$ is not affected by the wrapping effect (see Section 2.2.1).

In the following, the results of two of fault scenarios are showed in detail.

\section{Fault scenario 1: "Fixed value on Pitch 1 position sensor from $t=2000$ s to $t=2100$ s"}

Figure 1 and 2 show the nominal residuals $r_{2}^{0}, r_{4}^{0}, r_{5}^{0}$ and $r_{6}^{0}$ (obtained using (39) with $\xi=\xi^{0}$ and $n_{i}(k)=0$ ) and the observed fault signature components $\phi_{2}, \phi_{4}, \phi_{5}$ and $\phi_{6}$ obtained applying the consistency tests. The fault is detected at instant $\mathrm{t}=2000.04 \mathrm{~s}$ and as the only signature components activated are $\phi_{5}$ (permanently) and $\phi_{6}$ (intermittently), and the fault is isolated at the detection instant.
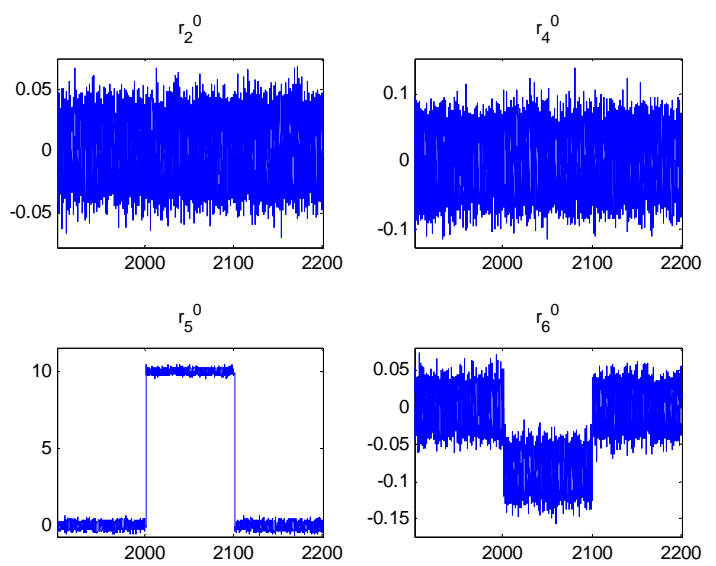

Fig. 6. Nominal residuals $r_{2}^{0}, r_{4}^{0}, r_{5}^{0}$ and $r_{6}^{0}$ in fault scenario 1 .
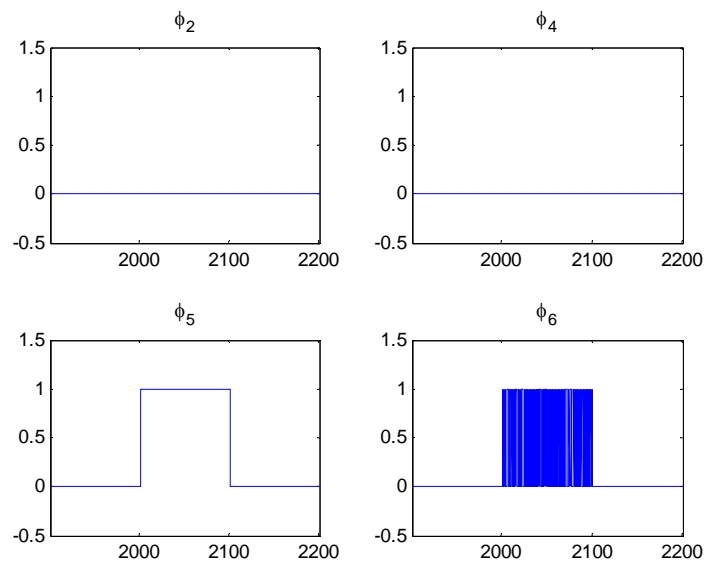

Fig. 7. Observed fault signature components $\phi_{2}, \phi_{4}, \phi_{5}$ and $\phi_{6}$ in fault scenario 1. 
Figure 3 and 4 show the nominal residuals $r_{2}^{0}, r_{3}^{0}, r_{4}^{0}$ and $r_{12}^{0}$ and the observed fault signature components $\phi_{2}, \phi_{3}, \phi_{4}$ and $\phi_{12}$. The fault is detected and isolated at instant $\mathrm{t}=1000.01 \mathrm{~s}$.
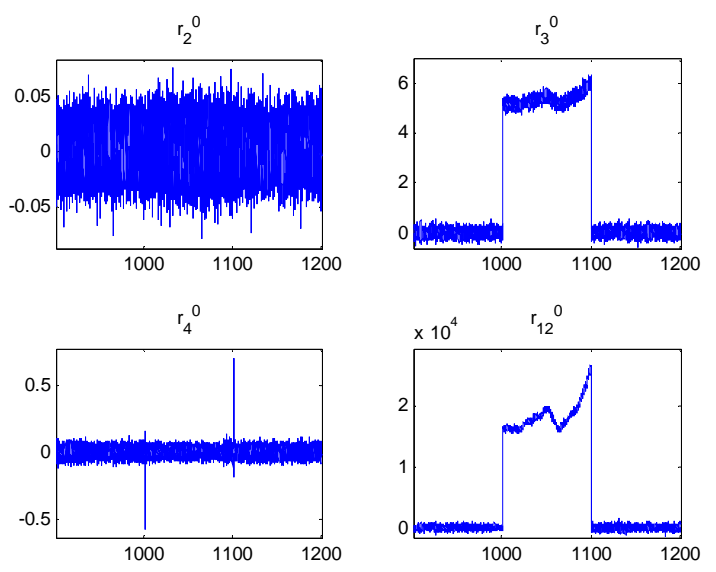

Fig. 8. Nominal residuals $r_{2}^{0}, r_{3}^{0}, r_{4}^{0}$ and $r_{12}^{0}$ in fault scenario 5 .
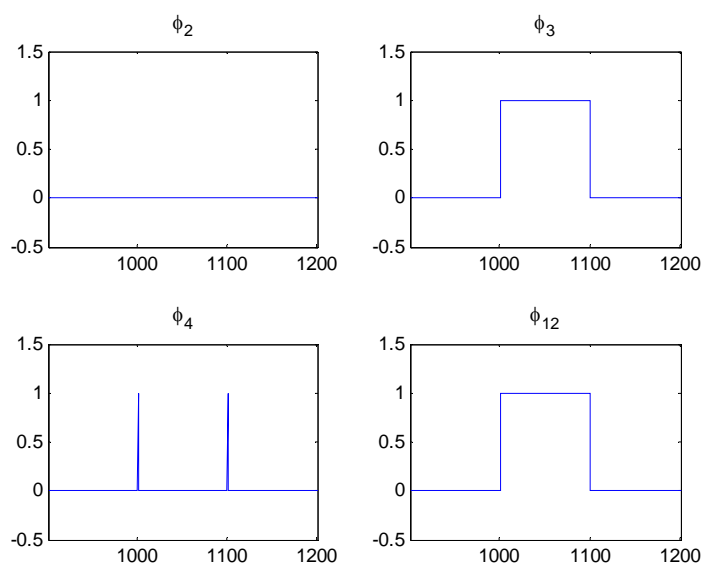

Fig. 9. Observed fault signature components $\phi_{2}, \phi_{3}, \phi_{4}$ and $\phi_{12}$ in fault scenario 5.

\section{CONCLUSIONS}

In this paper, a set-membership parity space approach for fault detection of linear uncertain dynamic systems has been proposed. This method obtains a set of parity relations derived from the parity space approach. Then, the parameter identification of those relations is performed using a set-membership algorithm. In particular, in this work the parametric uncertainty is bounded by a zonotope. Fault detection is based on checking, at every sampling time, the non existence of a parameter value in the parameter uncertainty set such that model is consistent with all the system measurements. The proposed approach has been applied to two case studies one based on two-tank system and another, more realistic, based in wind turbine FDI benchmark in order to evaluate its effectiveness providing satisfactory results. 


\section{ACKNOWLEDGEMENTS}

This work has been partially funded by the grant CICYT SHERECS DPI-2011-26243 and WATMAN DPI-2009-13744 of Spanish Ministry of Education.

\section{REFERENCES}

Basseville, M. and Nikiforov, I.V. (1993). Detection of Abrupt Changes - Theory and Application. Prentice-Hall, Inc. Englewood Cliffs, N.J.

Blanke, M., Kinnaert, M., Lunze, J., Staroswiecki, M. and Schröder, J. (2006a). Diagnosis and Fault-Tolerant Control. Springer-Verlag: New York.

Blanke M. and Lorentz T. (2006b) “SaTool: A software tool for structural analysis of complex automation systems”. In Proceedings of the 6th IFAC Symposium on Fault Detection, Supervision and Safety of Technical Processes, pages 673-678, Beijing, PR China., 2006.

Blesa, J., Puig, V., Saludes J. (2011a). Identification for passive robust fault detection using zonotope-based set-membership approaches. International Journal of Adaptive Control and Signal Processing. 25 (9): 788-812.

Blesa, J., Puig,V., Romera,J. and Saludes,J.(2011b).Fault diagnosis of windturbines using a set-membership approach. In Proceedings of the 18th IFAC worldcongress (IFAC). Milan,Italy. 28 August-2 September 2011 ; pp. 8316-8321.

Blesa, J., Puig, V., Saludes, J. (2012). "Robust Fault Detection using Polytope-based Set-membership Consistency Test". IET control theory and applications, 6 (12): 1767-1777.

Blesa, J., Rotondo, D., Puig, V., Nejjari, F. (2014). FDI and FTC of windcturbines using the interval observer approach and virtual actuators/sensors. Control Engineering Practice 24(2014) 138-155.

Bravo, J.M., Alamo, T. Camacho, E.F. (2006). Bounded error identification of systems with time-varying parameters. IEEE Transactions on Automatic Control, vol. 51, no. 7, pp. $1144-1150$.

Chen, J. and Patton, R.J. (1999). Robust Model-Based Fault Diagnosis for Dynamic Systems. Kluwer Academic Publishers: Dordrecht.

Chilali M, Gahinet P, Apkarian P. (1999) Robust pole placement in LMI regions. IEEE Transactions on Automatic Control; 44(12):22572270.

Chow, E.Y. and Willsky, A.S. (1984). Analytical redundancy and the design of robust failure detection systems. IEEE Transactions on Automatic Control 29, pp. 603-614.

Ding, S.X (2008) Model-based Fault Diagnosis Techniques Design Schemes, Algorithms, and Tools. Springer: Berlin.

Gertler, J. (1998). Fault Detection and Diagnosis in Engineering Systems. Marcel Dekker: New York.

Horak DT.(1988). Failure detection in dynamic systems with modelling errors. Journal of Guidance, Control, and Dynamics; 11(6):508-516.

Isermann, R. (2006). Fault-diagnosis systems: An introduction from fault detection to fault tolerance. Springer: Berlin.

Jaulin, L., Kieffer, M., Didrit, O., Walter E. (2001) Applied interval analysis. Springer.

Meseguer, J., Puig, V., Escobet, T. (2010). “Observer Gain Effect in Linear Interval Observer-based Fault Detection” Journal of Process Control Vol. 20, No. 8, pp. 944-956.

Milanese, M., Norton, J., Piet-Lahanier, H. and Walter E. (eds). (1996). Bounding Approaches to System Identification. Plenum Press: New York. 
Odgaard, P.F., Stoustrup, J. and Kinnaert, M. (2013). Fault tolerant control of wind turbines - a benchmark model. IEEE Control Systems Technology, Vol. 21, No. 4, pp. 1168-1182.

Ploix, S. and Adrot, A. (2006). Parity relations for linear uncertain dynamic systems. Automatica, 42:1553 1562.

Puig, V., Saludes, J., Quevedo, J. (2003) “Worst-Case Simulation of Discrete Linear Time-Invariant Interval Dynamic Systems”. Reliable Computing, 9(4), pp. 251-290.

Puig, V., Stancu, A., Quevedo, J. (2005) “Set versus Trajectory based Approaches to Interval Observation”. Conference on Decision and Control \& European Control Conference (CDC-ECC’05). Sevilla. Spain.

Puig, V., Ingimundarson, A., Tornil-Sin, S. (2006). "Robust Fault Detection using Inverse Images of Interval Functions”. 6th IFAC Symposium on Fault Detection, Supervision and Safety of Technical Processes (SAFEPROCESS 2006). Beijing, PR. China

Puig, V., Quevedo, J., Escobet, T., Nejjari, F. \& de las Heras, S. (2008). "Passive Robust Fault Detection of Dynamic Processes Using Interval Models”. IEEE Transactions on Control Systems Technology, 16(5), 1083 - 1089. Puig, V. (2010). “Fault diagnosis and fault tolerant control using set-membership approaches: Application to real case studies”. International journal of applied mathematics and computer science, 20(4), pp. 619-635

Tabatabaeipour, S. M. , Odgaard, P. F., Bak, T., Stoustrup J. (2012). "Fault detection of wind turbines with uncertain parameters: A setmembership approach." Energies, 5(7), 2424-2448.

Tabatabaeipour, S. M. (2013). “Active fault detection and isolation of linear time varying systems: a set-membership approach”. International Journal of Systems Science, DOI: 10.1080/00207721.2013.843213

Tornil-Sin, S., Ocampo-Martinez, C., Puig, V., Escobet, T. “Robust Fault Diagnosis of Nonlinear Systems Using Interval Constraint Satisfaction and Analytical Redundancy Relations”. IEEE Transactions on Systems, Man, and Cybernetics: Systems. To appear: DOI:10.1109/TSMC.2013.2238924

Vicino A. and Zappa G. (1996). Sequential aproximation of feasible parameter sets for identification with set membership uncertainty. IEEE Trans. Autom. Control, vol. 41, no. 6, pp. 774-785. 